Article

\title{
A Single Cell Extraction Chip Using Vibration-Induced Whirling Flow and a Thermo-Responsive Gel Pattern
}

\section{Takeshi Hayakawa $^{1, *}$, Shinya Sakuma ${ }^{1}$, Takeshi Fukuhara ${ }^{2}$, Yoshiyuki Yokoyama ${ }^{3}$ and Fumihito Arai ${ }^{1}$}

${ }^{1}$ Department of Micro-Nano Systems Engineering, Nagoya University, Furo-Cho, Chikusa-Ku, Nagoya 464-8601, Japan; E-Mails: sakuma@mech.nagoya-u.ac.jp (S.S.); arai@mech.nagoya-u.ac.jp (F.A.)

2 Laboratory of Oncology, School of Life Sciences, Tokyo University of Pharmacy and Life Sciences, 1432-1 Horinouchi, Hachiohji, Tokyo 192-0392, Japan; E-Mail: tftoyaku@gmail.com

3 Toyama Industrial Technology Center, 383 Takata, Toyama 930-0866, Japan;

E-Mail: yokoyama@itc.pref.toyama.jp

* Author to whom correspondence should be addressed;

E-Mail: t-hayakawa@biorobotics.mech.nagoya-u.ac.jp; Tel.: +81-54-789-5026.

Received: 17 July 2014; in revised form: 27 August 2014 / Accepted: 27 August 2014 /

Published: 10 September 2014

\begin{abstract}
We propose a single cell extraction chip with an open structure, which utilizes vibration-induced whirling flow and a single cell catcher. By applying a circular vibration to a micropillar array spiral pattern, a whirling flow is induced around the micropillars, and target cells are transported towards the single cell catcher placed at the center of the spiral. The single cell catcher is composed of a single-cell-sized hole pattern of thermo-responsive gel. The gel swells at low temperatures $\left(\lesssim 32{ }^{\circ} \mathrm{C}\right)$ and shrinks at high temperatures $\left(\gtrsim 32^{\circ} \mathrm{C}\right)$, therefore, its volume expansion can be controlled by an integrated microheater. When the microheater is turned on, a single cell is trapped by the hole pattern of the single cell catcher. Then, when the microheater is turned off, the single cell catcher is cooled by the ambient temperature. The gel swells at this temperature, and the hole closes to catch the single cell. The caught cell can then be released into culture wells on a microtiter plate by heating the gel again. We conducted single cell extraction with the proposed chip and achieved a $60 \%$ success rate, of which $61 \%$ cells yielded live cells.
\end{abstract}

Keywords: cell manipulation; single cell extraction; acoustic streaming; PNIPAAm 


\section{Introduction}

Recent progress in biotechnology and micro-/nano-technology has enabled single cell analysis, which reveals cell heterogeneity in an isogenic cell population $[1,2]$ and is expected to be applied to regenerative therapy and antibody medicine. To analyze these single cell characteristics, it is necessary to isolate a single cell from the original population to eliminate influences of other cells. Furthermore, to establish the monoclonal colony, which is cloned from an original single cell in isolation from other cells, is especially critical for quality-controlled mass-production in antibody medicine. Therefore, a technology to extract a single cell from the original population and separate it in an isolated area (e.g., a culture well on microtiter plate) is strongly needed.

Currently, fluorescent-activated cell sorters (FACSs) are widely used for extracting a single cell from a large number of cells in a high throughput manner [3,4]. FACSs employ many components to achieve this performance, such as pumps for cell transport, lasers for cell detection, a piezoelectric actuator for a single-cell-containing droplet generation and a high-voltage electric source for directing the cell onto a microtiter plate. This configuration of many components leads to a large system and requires huge initial costs. Therefore, microfluidic chip for a single cell extraction has been attracting considerable attention recently. One of the biggest advantages of the microfluidic chip is that it can integrate multiple functions into the same chip. In the case of a single cell extraction, a cell transport and extraction mechanism can be integrated on one chip, making the entire system simpler, smaller, and cheaper in terms of initial cost. Moreover, microfabrication technologies allow mass production of such chips, thereby meeting the single use requirement at clinical and biological laboratories to avoid contamination and infection.

Many researchers proposed on-chip single cell separation [5-11]. Most of this research employed precise fluid control in a closed channel as a single cell separation mechanism and cell transportation towards the separation mechanism. Although they succeeded in separating a single cell in a closed channel on the chip, a single cell extraction from the chip to an external environment has not been successful. This difficulty of extraction is not favorable for cloning and time lapse observation during single cell analysis. The biggest problem is extracting a single cell from a closed chip to an external environment. Using a closed chip, extracting a single cell is difficult because it is easily lost at an interface with the external environment, such as a tube connection. Therefore, an open-structure chip is suitable for a single cell extraction. However, by using an open-structure chip, we cannot utilize flow control in a closed channel as a single cell separation mechanism and cell transport. Thus, a single cell extraction mechanism and cell transport, which can be used even on an open-structure chip, are necessary to realize on-chip single cell extraction.

In this paper, we propose an open-structure chip to realize single cell extraction from a chip to an external environment. We employ vibration-induced whirling flow for cell transport, and a single cell catcher as the single cell extraction mechanism, which can be used even on an open-structure chip. The whirling flow is induced by applying circular vibration to micropillars on the chip and is utilized to gather target cells to the single cell catcher. The single cell catcher is composed of a single cell sized hole pattern of thermo-responsive gel and can catch and release only a single cell from original cell population by means of the swelling and shrinking of the gel. It is controlled by temperature change actuated by turning on and off a microheater fabricated on the chip. 
The basic concept is described in the second section, and the fabrication process is explained in the third section. Theoretical and experimental analyses are conducted, and the design of the chip is determined in the fourth section. Finally, we perform single cell extraction and summarize the study.

\section{Concept}

\subsection{Cell Transport by Vibration-Induced Whirling Flow}

To achieve single cell extraction with an open-structure chip, we have to implement a single cell extraction mechanism, which is described in detail in the next subsection, and a cell transport method to transport target cells to the mechanism. Some cell transport methods, such as optical tweezers [12-14], dielectrophoresis (DEP) [15-17] and surface acoustic wave (SAW) [18,19], can be applied to an open-structure chip. Using optical tweezers, a single cell can be manipulated with high positioning accuracy, but the manipulation area is small, approximately $100 \mu \mathrm{m} \times 100 \mu \mathrm{m}$. Supplying target cells to such a small area manually without any additional manipulators is difficult. DEP can be used for manipulating target cells in a large area by adjusting electrode geometry, but moving the direction and trajectory of cells by DEP is dependent on applied voltage, frequency and cell type. Therefore, different applied voltage and frequency conditions must be applied to different cell types, and this is time-consuming. SAW can utilize standing wave or SAW-induced flow to manipulate cells in both small and large areas. These manipulations are based on fluid force and are independent of the target cell type. However, SAW has to use piezoelectric materials as a substrate, and this leads to a high running cost compared with typical glass and silicon substrates.

Considering the features of the above methods, we focus our attention on a vibration-induced flow around an object on a chip. Many studies on a vibrating object in fluid and its effects on local flow induction were reported [20-23]. This phenomenon was confirmed even on an open-structure chip (Figure 1a). Figure 1a shows the streamline around an SU-8 (Nippon Kayaku Co. Ltd., Tokyo, Japan) micropillar visualized by long time exposure of fluorescent beads. This local whirling flow can be observed by applying circular vibration to the micropillar fabricated chip. The streamline is localized in the vicinity of the micropillar $(\simeq 50 \mu \mathrm{m})$, and the flow induction has good repeatability even on an open-structure chip. Furthermore, the flow velocity and direction can be modulated by changing the applied vibration frequency and direction. Therefore, this vibration-induced whirling flow can be applied for flow control and manipulation of cells on an open-structure chip.

Figure 2 depicts the basic concept of the single cell extraction chip. Cell transport is achieved by the micropillar array and a circular vibration. By applying a circular vibration to the chip, a local whirling flow is induced in the vicinity of each micropillar. If the micropillar pitch is shorter than the flow-induced area thickness, "global" whirling flow can be generated along the micropillar array. Thus, by adjusting the micropillar array pitch and configuration, we can transport target cells to an arbitrary position. For single cell extraction, we employ a spiral pattern (diameter $6 \mathrm{~mm}$ ) to gather target cells from all directions over a large area towards the center of the spiral. The proposed chip can be used for any type of cell, and it is manufactured from a normal glass substrate with low material cost. 
Figure 1. Microscopic image of basic evaluation: (a) vibration-induced local whirling flow around the micropillar, (b) single cell catching with patterned thermo-responsive gel (bioresist).

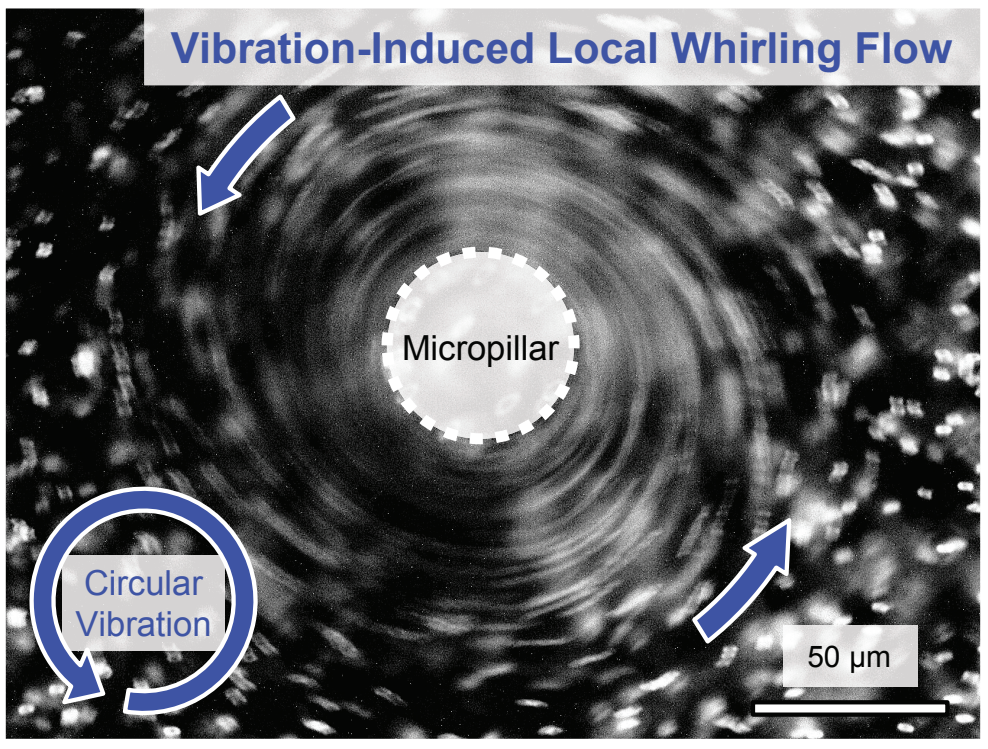

(a)

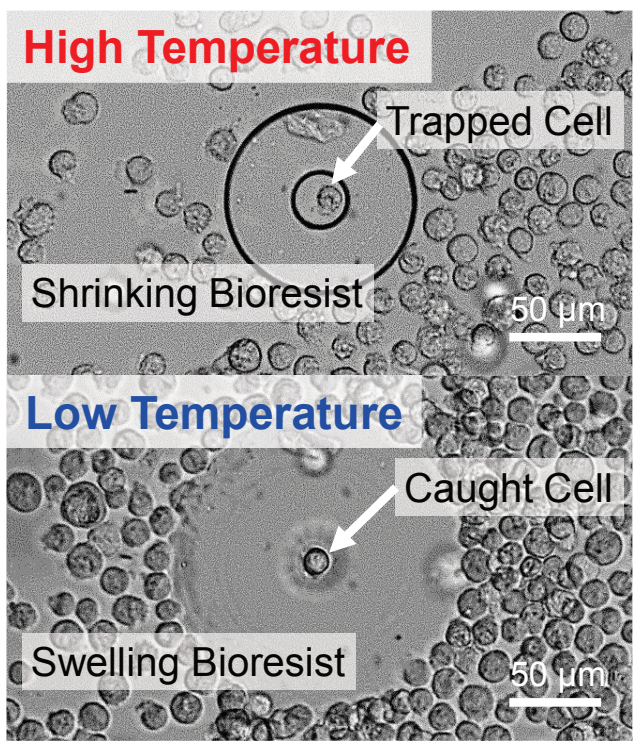

(b)

Figure 2. Basic concept of the single cell extraction chip.

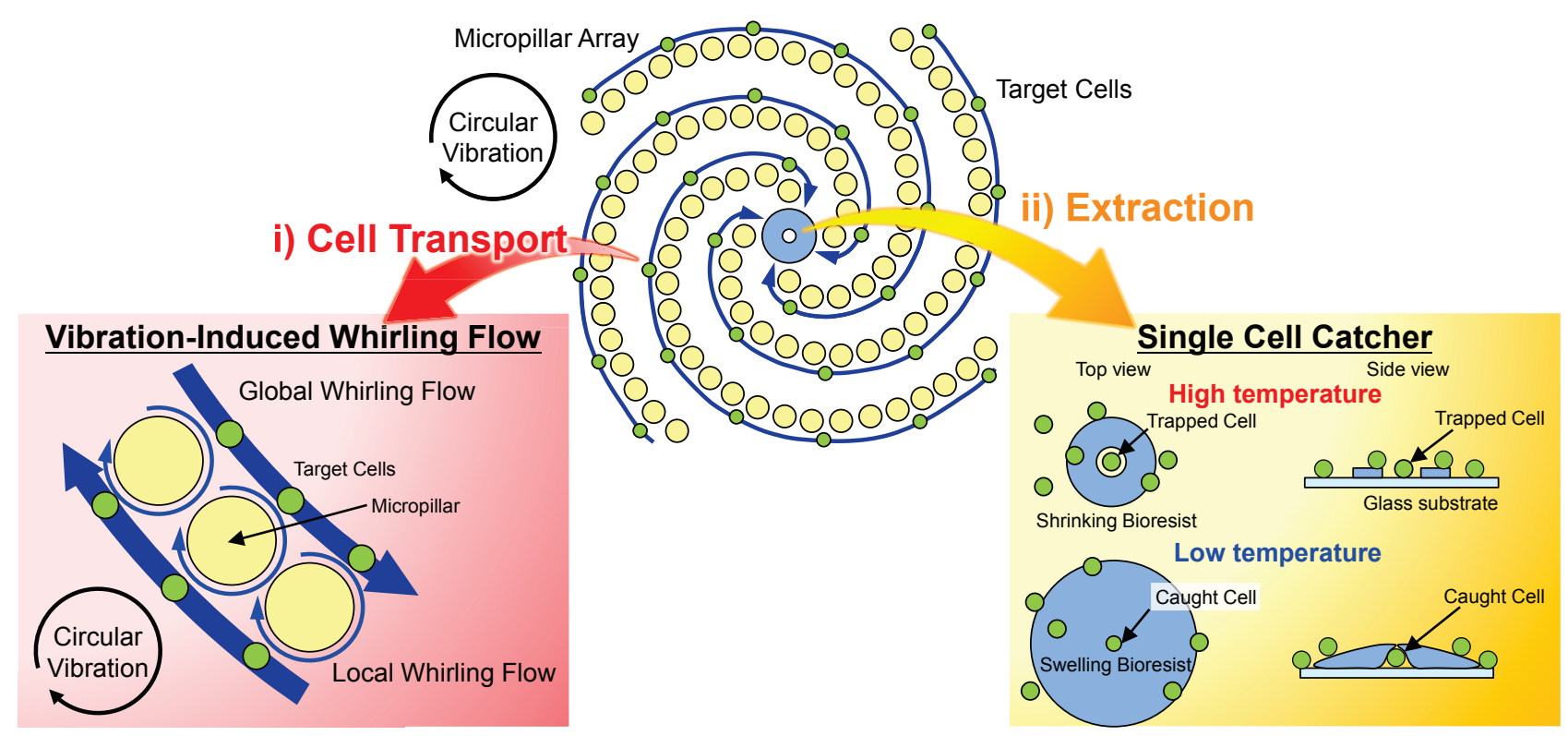

\subsection{Single Cell Catcher by Thermo-Responsive Gel Pattern}

To extract a single cell, we must first separate a single cell from the original cell population. Then, by removing other cells and transferring the separated single cell to an isolated area, such as a culture well on a microtiter plate, the single cell extraction is achieved. We employ a single cell catcher composed of photoprocessable thermo-responsive gel (bioresist) (Nissan Chemical Industries, Ltd., Tokyo, Japan) [24,25] as an extraction mechanism to achieve this separation and transfer (Figure 1b). The bioresist can be patterned by a photolithography process, and it shrinks at high temperature $\left(\gtrsim 32{ }^{\circ} \mathrm{C}\right)$, 
and swells at low temperature $\left(\lessgtr 32^{\circ} \mathrm{C}\right)$. Therefore, by dropping target cells into a single cell sized hole pattern of the bioresist under heating, a single cell can be trapped in the hole. When we turn off heating and the single cell catcher cools below $\approx 32{ }^{\circ} \mathrm{C}$, the trapped single cell is securely caught by the swelling bioresist. Other cells can be removed by washing the chip while the cell is caught by the single cell catcher. Then, the single cell catcher is shrunk again by heating, and the caught cell can be released into an external culture well by opening the hole. Therefore, single cell extraction can be achieved using the single cell catcher.

\subsection{Concept of Single Cell Extraction System}

The basic concept of the single cell extraction chip is depicted in Figure 3. The system consists of a single cell extraction chip with an open structure, an actuator for applying circular vibration and a microscope for observation. The micropillar spiral pattern, single cell catcher and microheater are integrated on the single cell extraction chip. In this study, circular vibration is applied to the chip by independent XY piezoelectric actuators, and the single cell catcher is heated by the microheater on the chip.

Using this system, a single cell extraction can be achieved by the following steps:

(i) Transport: Drop a sample cell suspension on the chip, and apply circular vibration (Figure 3b). Circular vibration induces a global whirling flow directed towards the center of the spiral, and the dropped cells are transported there. By turning on the microheater, the hole of the single cell catcher is opened. When the single cell transported to the center of the hole is detected by the microscope, the circular vibration is turned off and the single cell is trapped in the hole.

(ii) Catch: Turn off the microheater to swell the single cell catcher. The single cell catcher is cooled by the ambient temperature, and the trapped cell is caught by the swelled single cell catcher. Then, cells that have not been caught are removed by washing the chip with phosphate buffered saline.

(iii) Release: Prepare a microtiter plate and fill the wells on the plate with culture medium. Put the inverted single cell extraction chip on the wells and turn on the microheater (Figure 3c). The single cell catcher is shrunk again by the heating, and the caught cell is released into the well. Thus, the single cell extraction is achieved by the proposed chip.

In this study, we connected two independent XZ piezoelectric actuators (AE0505D08DF, MESS-TEK Co., Ltd., Saitama, Japan) to a chip mount (see the experimental setup in Figure 3b). The single cell extraction chip is fixed by adhesive on the chip mount and circular vibrations were created by applying sinusoidal input signals to piezoelectric actuators with 90 or 270 degrees phase difference. All applied voltage in this study is $100 \mathrm{~V}$ and the actual vibration amplitude was $4.7 \mu \mathrm{m}$, which was measured at $200 \mathrm{~Hz}$ by high-speed camera (VW-6000, Keyence Corporation, Osaka, Japan).

Since most functions for single cell extraction are integrated on the chip, the entire system is simplified, free of any pumps, lasers or micromanipulators. Furthermore, the set of the micropillar array, bioresist and microheater can be arrayed on the chip. Thus, we can adjust the geometry of the set to match the geometry of the culture well with that of the microtiter plate, and parallel single cell extraction can be performed simultaneously. 
Figure 3. Concept of the single cell extraction system: (a) concept of the system and procedure, (b) experimental photograph of sample cell suspension loading, (c) experimental photograph of cell transfer.

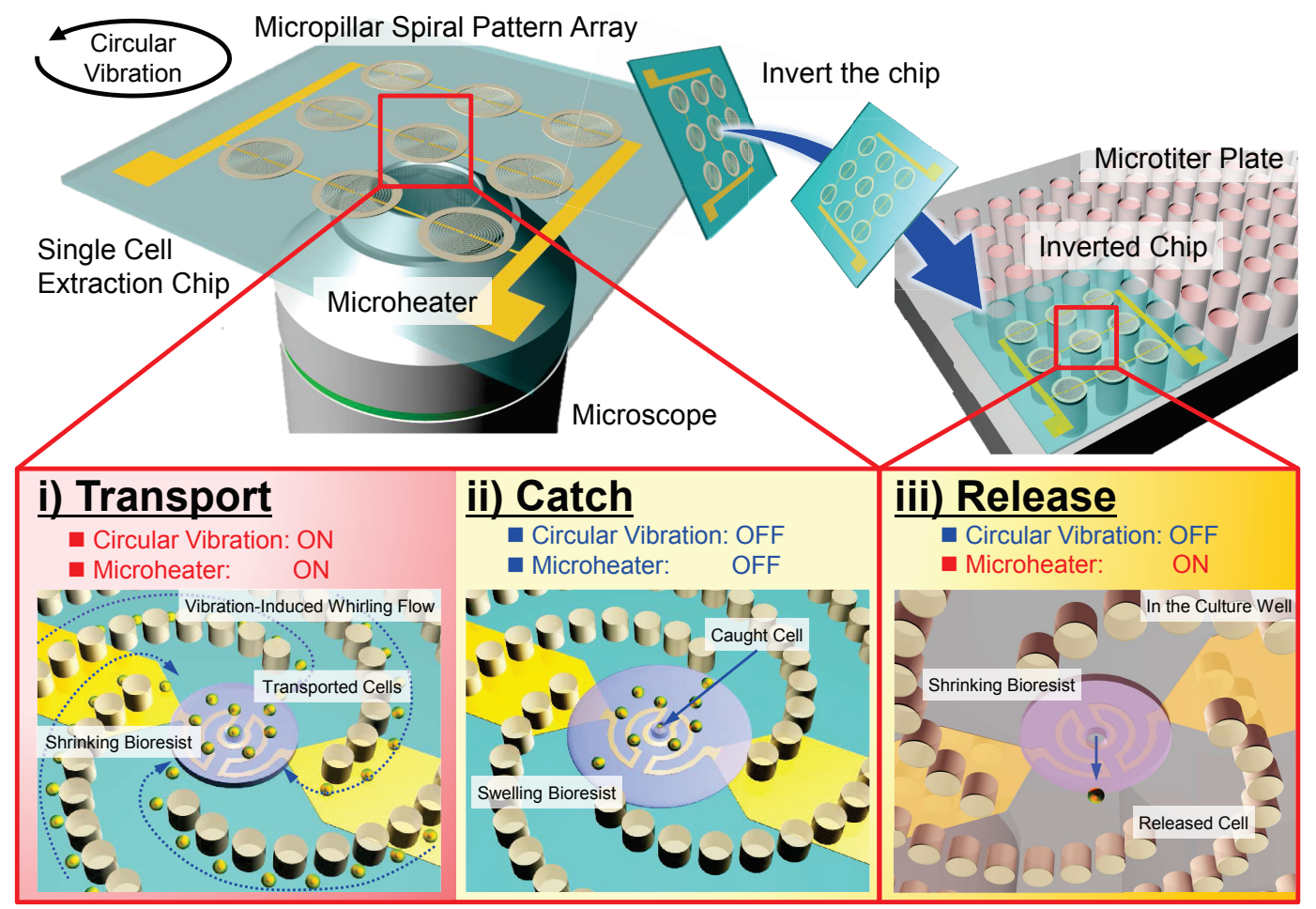

(a)

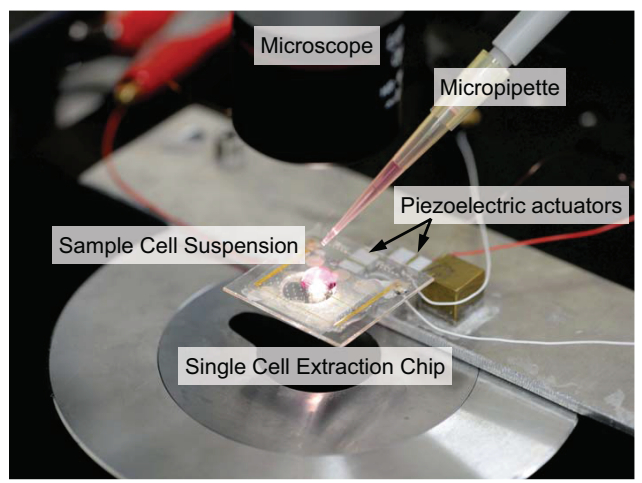

(b)

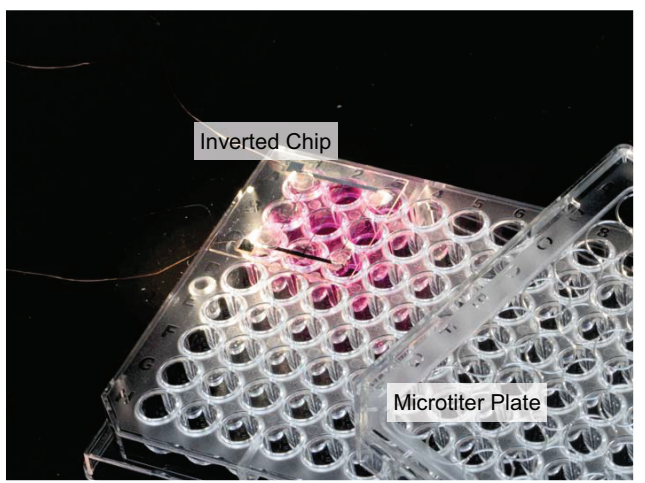

(c)

\section{Fabrication Process}

The fabrication process of the single cell extraction chip is shown in Figure 4a. The process consists of microheater patterning, bioresist patterning, and micropillar patterning. The detailed process flow is explained as follows.

1. Sputtering of $\mathrm{Cr} / \mathrm{Au}$ on a glass substrate.

2. Spin coating of OFPR (Tokyo Ohka Kogyo Co. Ltd., Tokyo, Japan) on the sputtered Cr/Au layer.

3. Exposure and development of the OFPR as an etching mask of the microheater. 
4. Etching $\mathrm{Cr} / \mathrm{Au}$ and removal of the OFPR.

5. Spin coating the bioresist on the patterned microheater.

6. Exposure and development of the bioresist as a single cell catcher.

7. Spin coating SU-8.

8. Exposure and development of SU-8 as a micropillar array.

A fabricated chip photograph and scanning electron microscope (SEM) image are shown in Figure $4 b, c$, respectively, demonstrating that we succeeded in fabricating the proposed chip.

Figure 4. Fabrication of the proposed open-structure chip: (a) fabrication process flow, (b) photograph of fabricated chip, (c) scanning electron microscope (SEM) image of fabricated chip.

1) Sputtering $\mathrm{Cr} / \mathrm{Au}$

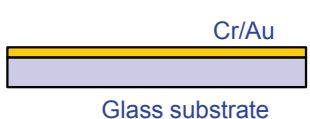

5) Spin coating Bioresist

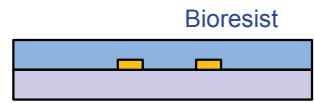

2) Spin coating OFPR

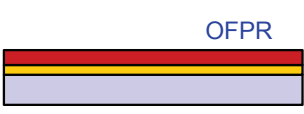

6) Exposure \& Development

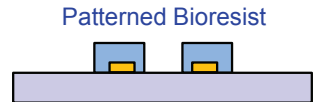

3) Exposure \& Development

Patterned OFPR

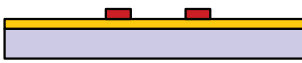

7) Spin coating SU-8

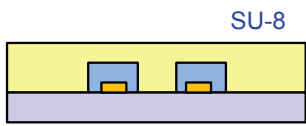

4) Etching $\mathrm{Cr} / \mathrm{Au}$ \& Removal of OFPR

Patterned Electrode

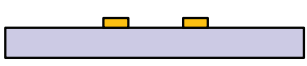

8) Exposure \& Development

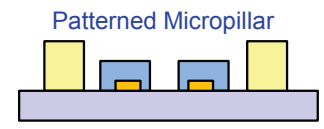

(a)

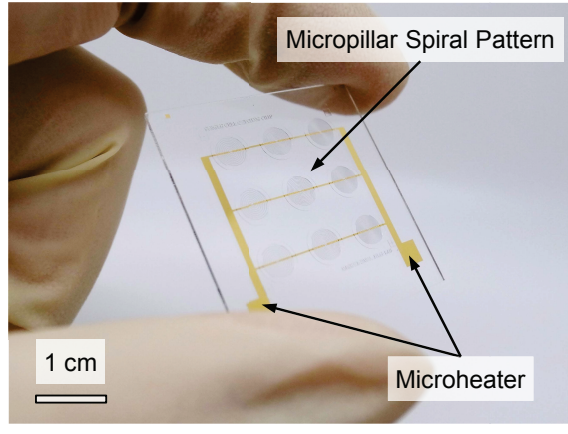

(b)

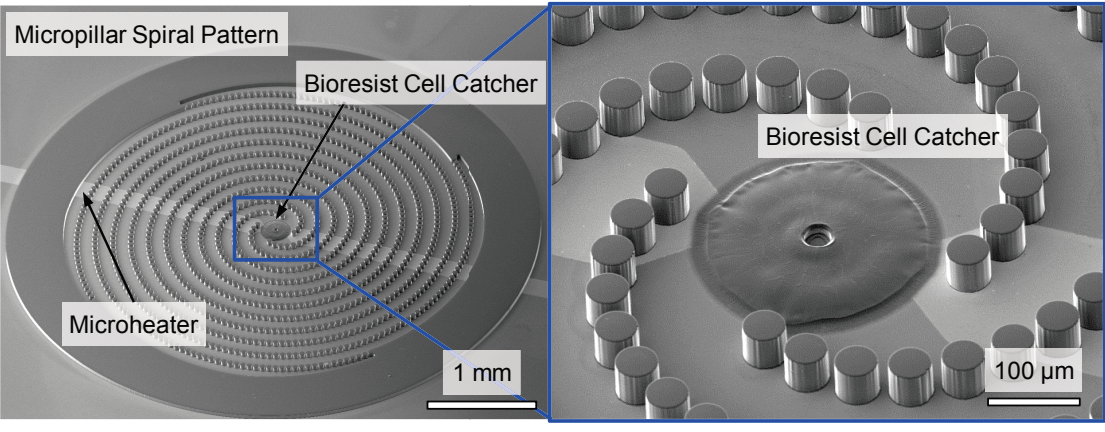

(c)

\section{Analysis and Design}

\subsection{Analysis of Vibration-Induced Local Whirling Flow}

To design a micropillar array pattern, we analyze the vibration-induced local whirling flow phenomenon theoretically. Many theoretical and experimental studies on vibrating obstacles in fluids were reported [20,21], and recently, some applications on a chip were proposed [22,23]. In this paper, we employ the model used in Holtsmark's study [20], but apply boundary conditions of circular vibration that are dissimilar from those in his study for single axis vibration. 
The basic differential equations for the motion of an incompressible viscous fluid in two-dimensional space can be written as

$$
\begin{gathered}
\nabla^{4} \psi-\frac{1}{\eta} \frac{\partial}{\partial t} \psi==\frac{1}{\eta} \mathbf{v} \cdot \nabla\left(\nabla^{2} \psi\right)=\frac{u}{\eta} \frac{\partial}{\partial x} \nabla^{2} \psi+\frac{v}{\eta} \frac{\partial}{\partial y} \nabla^{2} \psi \\
\frac{\partial u}{\partial x}+\frac{\partial v}{\partial y}=0
\end{gathered}
$$

Here, $\psi$ is a stream function, $\eta$ is the kinematic viscosity, $\mathbf{v}$ is the velocity vector, $u$ and $v$ are the velocity components in the $x$ and $y$ directions, respectively. We first solve the equation by assuming that the convection term in the right side is smaller than other terms.

$$
\nabla^{4} \psi^{(0)}-\frac{1}{\eta} \frac{\partial}{\partial t} \psi^{(0)}=0
$$

$\psi^{(0)}$ indicates the solution of zeroth order Equation (3). Under a circular vibration $A_{0}[\cos \omega t \hat{\mathbf{x}}+\cos (\omega t \pm \pi) \hat{\mathbf{y}}]$, the boundary conditions at the surface of the micropillar and at infinite distance from the micropillar can be written as follows (see the coordinate system setting in Figure 5a). We assume that the fluid obeys applied vibration at an infinite distance from the micropillar.

$$
\begin{aligned}
\left.v_{r}\right|_{r=a} & =\left.v_{\theta}\right|_{r=a}=0 \\
\left.v_{r}\right|_{r \rightarrow \infty} & =\omega A_{0}[\cos \theta \cos \omega t+\sin \theta \cos (\omega t \pm \pi)] \\
\left.v_{\theta}\right|_{r \rightarrow \infty} & =-\omega A_{0}[\sin \theta \cos \omega t-\cos \theta \cos (\omega t \pm \pi)]
\end{aligned}
$$

In these equations, $v_{r}$ and $v_{\theta}$ are the velocity components in the $r$ and $\theta$ directions, respectively, $a$ is the radius of the micropillar, $A_{0}$ is the amplitude of vibration and $\omega$ is the angular frequency of the vibration (Figure 5a). The positive (negative) sign of the phase indicates anticlockwise (clockwise) circular vibration. Solving this zeroth order equations, we acquire the zeroth order solution.

$$
\psi^{(0)}=\omega A_{0} a\left[\sin \theta e^{-i \omega t}-\cos \theta e^{-i(\omega t \pm \pi)}\right]\left(\frac{2 Y}{\epsilon}-\frac{r}{a}-\frac{a}{r} C\right)+\text { Complex Comjugate }
$$

Hereafter, we introduce $X, Y, Z$ and $C$ as abbreviations of radial functions expressed by first kind Hankel functions $H_{0}^{(1)}, H_{1}^{(1)}, H_{2}^{(1)}$.

$$
\begin{gathered}
X=\frac{H_{0}^{(1)}(\epsilon r)}{H_{0}^{(1)}(\epsilon a)}, \quad Y=\frac{H_{a}^{(1)}(\epsilon r)}{H_{0}^{(1)}(\epsilon a)}, \quad Z=\frac{H_{2}^{(1)}(\epsilon r)}{H_{0}^{(1)}(\epsilon a)}, \quad C=\frac{H_{2}^{(1)}(\epsilon a)}{H_{0}^{(1)}(\epsilon a)} \\
\epsilon=\left(i \frac{\omega}{\eta}\right)^{\frac{1}{2}}
\end{gathered}
$$

Then, we use the method of successive approximation to treat the nonlinear convection term in Equation (1). By substituting zeroth order solution into the right side of Equation (1), the first order equation can be acquired.

$$
\nabla^{4} \psi^{(1)}-\frac{1}{\eta} \frac{\partial}{\partial t} \psi^{(1)}=\frac{1}{\eta} \mathbf{v}^{(\mathbf{0})} \cdot \nabla\left(\nabla^{2} \psi^{(0)}\right)
$$


By performing some calculations, we acquire the following equations of the first order approximation of the stream function $\psi^{(1)}$ [20].

$$
\begin{aligned}
\left(\nabla^{4}-\frac{1}{\eta} \frac{\partial}{\partial t} \nabla^{2}\right) \psi^{(1)} & = \pm \rho(r) \pm 2 \mathrm{e}^{-2 i \omega t}(\cos 2 \theta \pm \sin \theta) \Omega(r) \pm 2 \mathrm{e}^{2 i \omega t}(\cos 2 \theta \mp \sin 2 \theta) \Omega^{\star}(r) \\
\rho(r) & =\frac{\omega^{3} A_{0}^{2}}{4 \eta^{2}}\left[2 X+2 X^{\star}-2 \frac{a^{2}}{r^{2}} C Z^{\star}-2 \frac{a^{2}}{r^{2}} C^{\star} Z-4 X X^{\star}+4 Z Z^{\star}\right] \\
\Omega(r) & =\frac{\omega^{3} A_{0}^{2}}{4 \eta^{2}}\left[Z-\frac{a^{2}}{r^{2}} C X\right]
\end{aligned}
$$

The upper sign in Equation (11) corresponds to the case of phase $+\pi$, and the lower sign corresponds to the case of phase $-\pi$. The first term on the right side of Equation (11) expresses steady flow, whereas others express oscillating flow. We only treat the steady term afterwards because we are now interested in steady flow that contributes to long term phenomena, such as cell transportations. By solving Equation (11), we obtain the steady term of the stream function $\psi_{s t}^{(1)}$.

$$
\begin{aligned}
\psi_{s t}^{(1)}= & \pm\left[r^{4}\left(\frac{1}{48} \int_{a}^{r} \frac{1}{x} \rho(x) d x+c_{1}\right)+r^{2}\left(-\frac{1}{16} \int_{a}^{r} x \rho(x) d x+c_{2}\right)\right. \\
& \left.+\left(\frac{1}{16} \int_{a}^{r} x^{3} \rho(x) d x+c_{3}\right)+\frac{1}{r^{2}}\left(-\frac{1}{48} \int_{a}^{r} x^{5} \rho(x) d x+c_{4}\right)\right] \\
c_{1}= & -\frac{1}{48} \int_{a}^{\infty} \frac{1}{x} \rho(x) d x, \quad c_{2}=\frac{1}{16} \int_{a}^{\infty} x \rho(x) d x \\
c_{3}= & \frac{a^{4}}{16} \int_{a}^{\infty} \frac{1}{x} \rho(x) d x-\frac{a^{2}}{8} \int_{a}^{\infty} x \rho(x) d x \\
c_{4}= & -\frac{a^{6}}{24} \int_{a}^{\infty} \frac{1}{x} \rho(x) d x+\frac{a^{4}}{16} \int_{a}^{\infty} x \rho(x) d x
\end{aligned}
$$

From these equations and the relationship between the stream function and velocity

$$
v_{r}=-\frac{1}{r} \frac{\partial \psi}{\partial \theta}, \quad v_{\theta}=\frac{\partial \psi}{\partial r}
$$

we plotted the velocity around a micropillar of radius $a=25 \mu \mathrm{m}$ with various frequencies (Figure $5 \mathrm{c}-\mathrm{e}$ ). The applied vibration is anticlockwise, amplitude $A_{0}=5 \mu \mathrm{m}$, and frequency is $f=50,100$, or 200 Hz. All numerical calculations were conducted using MATLAB R2012a (MathWorks Inc., Natick, MA, USA).

From Equation (12), the maximum velocity is strongly dependent on the frequency $\omega$. This is confirmed numerically and experimentally, as shown in Figure 5b. The experimental values were obtained by particle tracking of $10 \mu \mathrm{m}$ polystyrene microbeads. The experimental results are qualitatively in agreement with the theoretical results. However, when the applied frequency increases, the maximum velocity of the experimental result becomes lower than its theoretical value because of the use of finite-size microbeads. The calculated theoretical values are only for the stream velocity and do not consider the fluid resistance that the finite-size microbeads must receive. Therefore, the experimental values are lower than the theoretical ones. The difference becomes more obvious when the velocity increases. However, considering cell transport by using vibration-induced flow, this experimental value with $10 \mu \mathrm{m}$ microbeads, similar in size to target cells, is suitable for basic analysis of the design. 
Figure 5. Analysis of vibration-induced whirling flow: (a) coordinate settings, (b) frequency dependence of maximum velocity of theoretical and experimental results, (c) theoretical plot of absolute velocity distribution at $f=50 \mathrm{~Hz},(\mathbf{d}) f=100 \mathrm{~Hz}$, (e) $f=200 \mathrm{~Hz}$. Black arrows in $(\mathbf{c})-(\mathbf{e})$ indicate the flow direction.

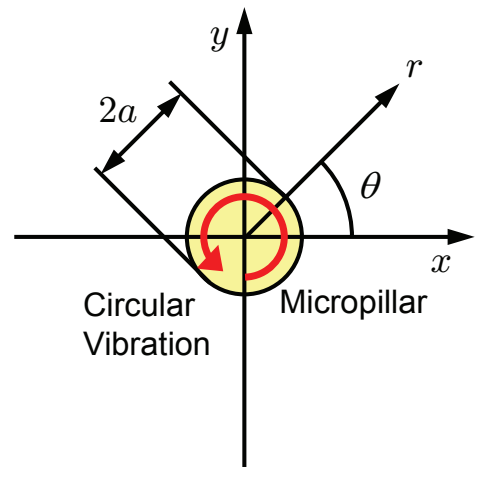

(a)

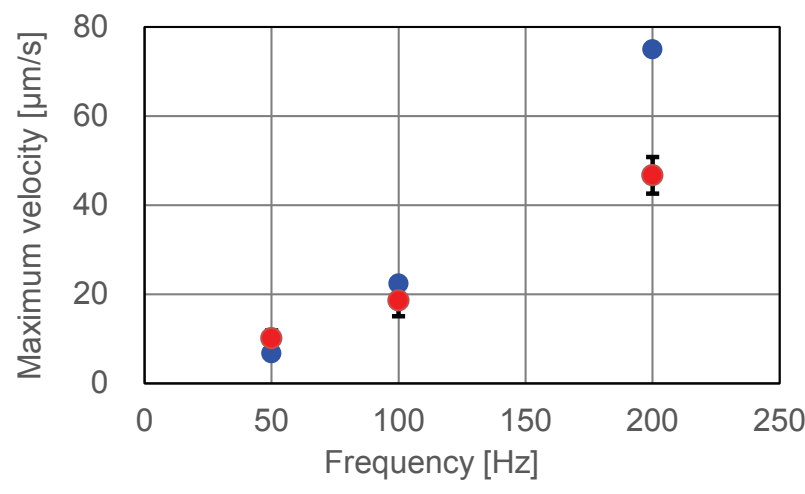

(b)

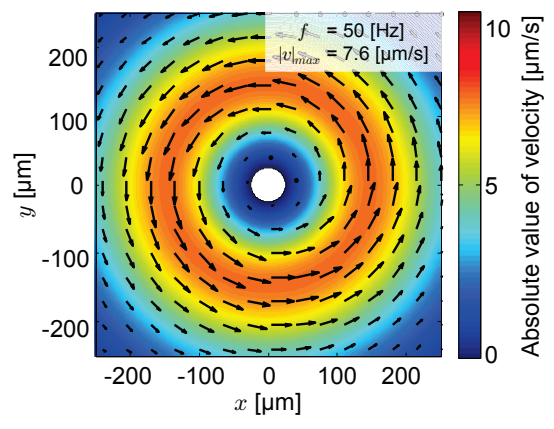

(c)

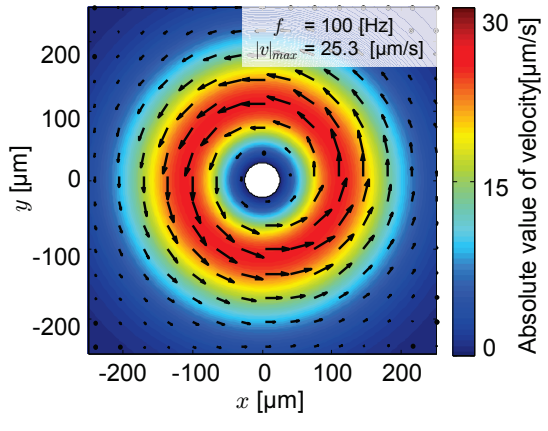

(d)

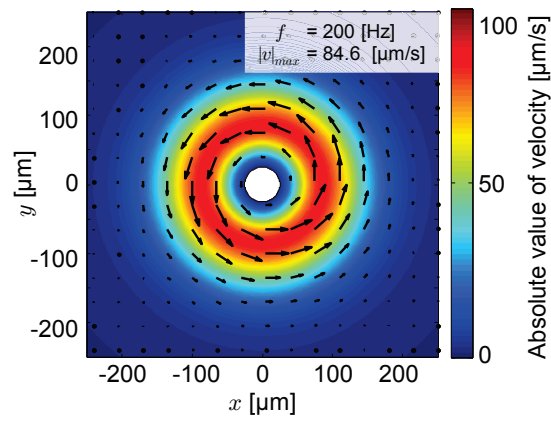

(e)

Additionally, flow-induced areas differ widely by frequency. This area can be estimated as a boundary layer thickness, $\delta \approx(\eta / \omega)^{1 / 2}$. Although higher frequency is favorable for faster cell transport, these boundary layer thicknesses become thinner as the transport area becomes smaller. Therefore, we have to design a micropillar array configuration while considering the applicable frequency and boundary layer thickness, as we will describe in next subsection.

Furthermore, the double sign in Equation (14) indicates that the sign of the stream function as well as the direction of the induced flow can be controlled by the applied vibration direction.

From theoretical and experimental studies, we have confirmed that vibration-induced whirling flow can readily control flow velocity and direction. Therefore, it can be used for flow control and cell transport on the proposed open-structure single cell extraction chip.

\subsection{Design of the Micropillar Array for Cell Transport}

From the results of the theoretical and experimental studies, we determine the design of the spiral pattern of the micropillar array for cell transport. As shown in Figure 6a, the design parameters of the micropillar array are pillar diameter $(2 a)$, pillar pitch $(P)$, transport channel width $(W)$ and pillar height $(H)$. In this study, a Madin-Darby canine kidney (MDCK) cell, with a size varying from 10 to $20 \mu \mathrm{m}$, is used as the target cell. 
Figure 6. Streamline of vibration-induced flow around a micropillar array: (a) Design parameters, and the microscopic image of streamline at (b) $\mathrm{W}=60 \mu \mathrm{m}$, (c) $\mathrm{W}=120 \mu \mathrm{m}$ and (d) $\mathrm{W}=180 \mu \mathrm{m}$. White arrows in the figure indicate flow directions.

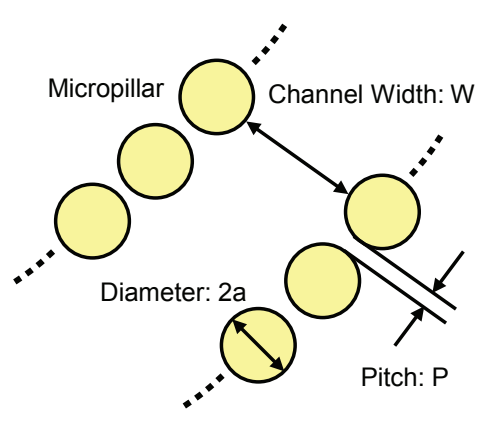

(a)

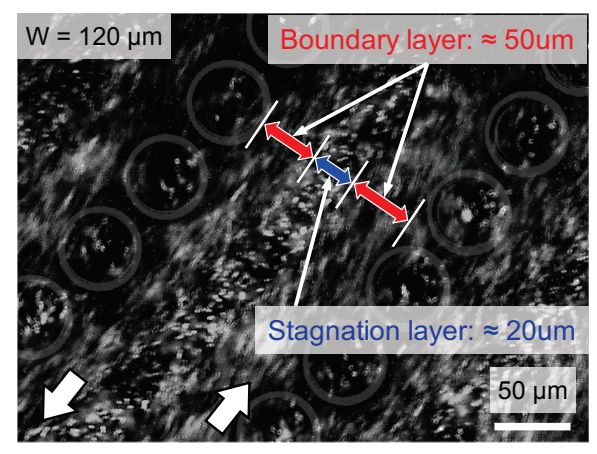

(c)

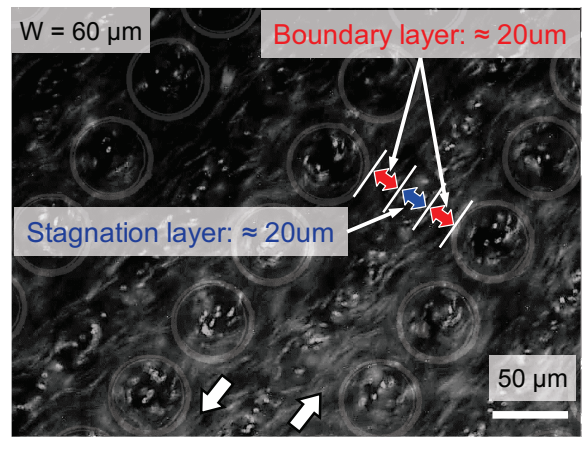

(b)

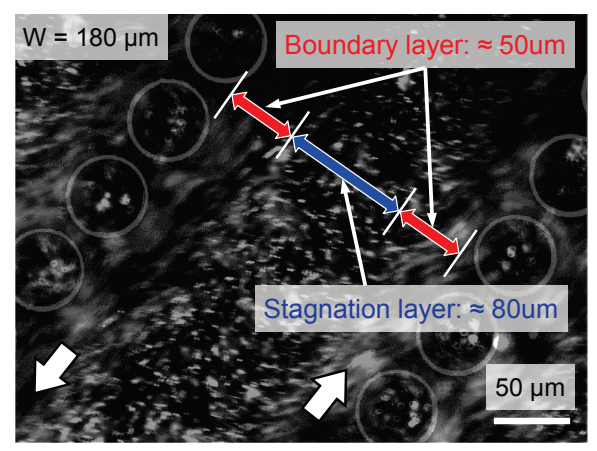

(d)

First, we determine the micropillar height $H=50 \mu \mathrm{m}$ and pitch $P=10 \mu \mathrm{m}$, so that target cells with size 10-20 $\mu \mathrm{m}$ can be easily transported and do not travel in between micropillars. From Equation (12), micropillar radius $a$ does not strongly affect the flow velocity compared with the distance from the micropillar surface. Therefore, we determine the micropillar radius $a=25 \mu \mathrm{m}$ (aspect ratio $=1.0$ ) by considering the processability. The most important parameter is the channel width $W$, and we determine it experimentally as follows.

In the present system, circular vibration can be stably applied up to $200 \mathrm{~Hz}$. We use this frequency for faster cell transport, and the boundary thickness is $\delta \approx 60 \mu \mathrm{m}$ at this frequency. Considering this value, channel width $W=120 \mu \mathrm{m}$ is thought to be favorable for cell transport because the entire channel area is occupied by vibration-induced flow. Narrower or wider channels are not good due to interference with the flow-induced area or inability to cover the channel center by the induced flow. We experimentally confirm this by varying the channel widths at $W=60(\delta), 120(2 \delta)$ and $180(3 \delta) \mu \mathrm{m}$. Figure $6 \mathrm{~b}-\mathrm{d}$ indicate the streamline in the channel of widths $W=60,120$ and $180 \mu \mathrm{m}$, respectively, visualized by long-term exposure of fluorescent beads. In Figure $6 b, c$, the flow velocity at the center of the channel is approximately zero because of the interference of the flow from both sides of the channel. The stagnation layer thickness is approximately $20 \mu \mathrm{m}$ for both cases. For $W=180 \mu \mathrm{m}$ (Figure 6d), the flow velocity at the center of the channel is approximately zero similar to the other $W$. However, the stagnation layer thickness is approximately $100 \mu \mathrm{m}$, which is larger than the case of other $W$ because the vibration-induced flow cannot cover such a wide channel. Our expectation is confirmed experimentally, and we set $W=120 \mu \mathrm{m}$ to obtain a wider transport channel. 


\subsection{Design of the Single Cell Catcher}

Next, we determine the design of the single cell catcher. The design parameters of the single cell catcher are a hole diameter $D$ and pattern width $W_{b}$ (Figure 7a). Hole diameter $D$ is determined so as to catch only a single cell. Since the target cell size is $10-20 \mu \mathrm{m}$, the diameter is determined to be $25 \mu \mathrm{m}$. Pattern width $W_{b}$ is related to the expansion rate of the cell catcher, as shown in Figure $7 \mathrm{~b}$. The wider the pattern width becomes, the more the expansion rate increases. On the other hand, the single cell catcher is integrated on a chip with the micropillar array. Thus, a very large single cell catcher is not favorable, as it results in patterning interference with the micropillars. Therefore, we evaluate the temperature characteristics of the expansion of single cell catchers with various pattern widths. Figure $7 \mathrm{c}$ shows the temperature characteristics of single cell catchers with $W_{b}=10,30,50$ and $100 \mu \mathrm{m}$. Figure $7 \mathrm{c}$ indicates that the single cell catchers with $W_{b}=10$ and $30 \mu \mathrm{m}$ are not completely closed, even at $20{ }^{\circ} \mathrm{C}$. On the other hand, the cell catcher with $W_{b}=100 \mu \mathrm{m}$ is completely closed at $27^{\circ} \mathrm{C}$, which is higher than typical room temperature. This feature is suitable for single cell extraction at room temperature. Therefore, we determined the pattern width to be $W_{b}=100 \mu \mathrm{m}$.

Figure 7. Single cell catcher: (a) design parameters, (b) expansion rate difference at different widths $W_{b}$ and (c) temperature characteristics of the single cell catcher with different pattern widths.

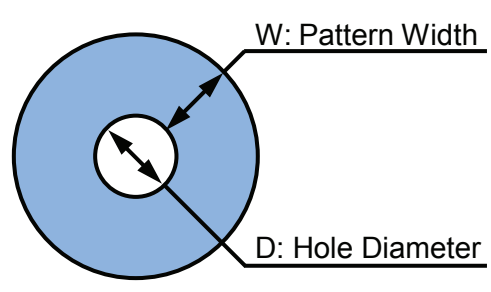

(a)

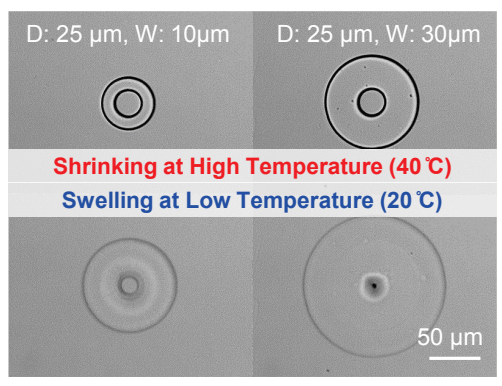

(b)

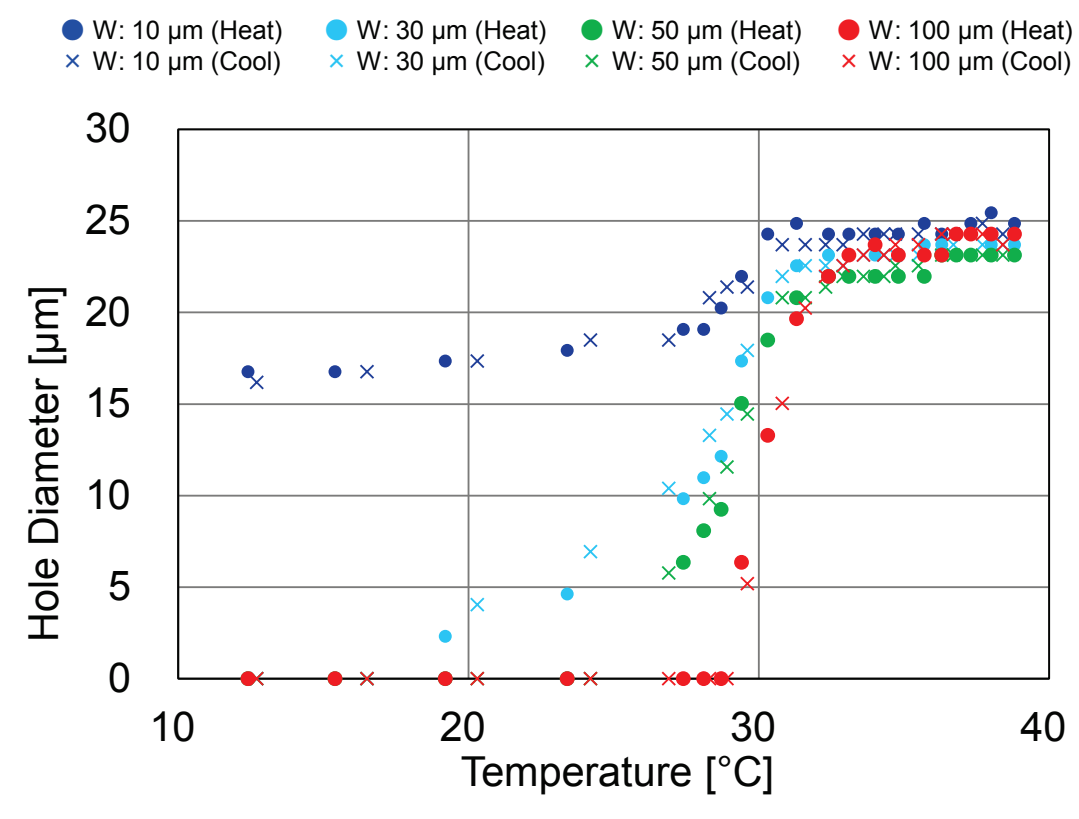

(c)

\section{Results and Discussion}

Finally, we perform single cell extraction using the proposed chip. Target cells were cultured on cell culture dish (Nunclon, Thermo Fisher Scientific K.K., Yokohama, Japan) with Dulbecco minimum essential medium eagle (EMEM) with $10 \%$ fetal bovine serum (FBS). These cells were treated with trypsin to acquire a cell suspension just before the experiments. Concentration of the cell suspension was adjusted to $5 \times 10^{3}$ cells $/ \mathrm{mL}$ and stored at $4{ }^{\circ} \mathrm{C}$ during experiments. $100 \mu \mathrm{L}$ samples were dropped 
onto the chip by micropipette in each trial. All processes of the single cell extraction were conducted at room temperature.

Target cell transport over $10 \mathrm{~s}$ with a $1 \mathrm{~s}$ time period between successive images is shown in Figure $8 \mathrm{a}$. Target cells are transported along the spiral pattern of the micropillar array towards the single cell catcher at the center of the spiral. The transported cells are gathered on the single cell catcher. Once the microscope detects a single cell in the hole of the bioresist cell catcher, the circular vibration is turned off, and the single cell is trapped by the hole (upper part of Figure $8 \mathrm{~b}$ ). Then, the microheater is turned off, and the swelling bioresist successfully catches the single cell (lower part of Figure 8b).

Figure 8. Microscopic image of on-chip single cell extraction: (a) successive picture of cell transport, (b) single cell catch by the single cell catcher, (c) confocal microscope image of the single cell catcher with cells before the catch and (d) after the catch.

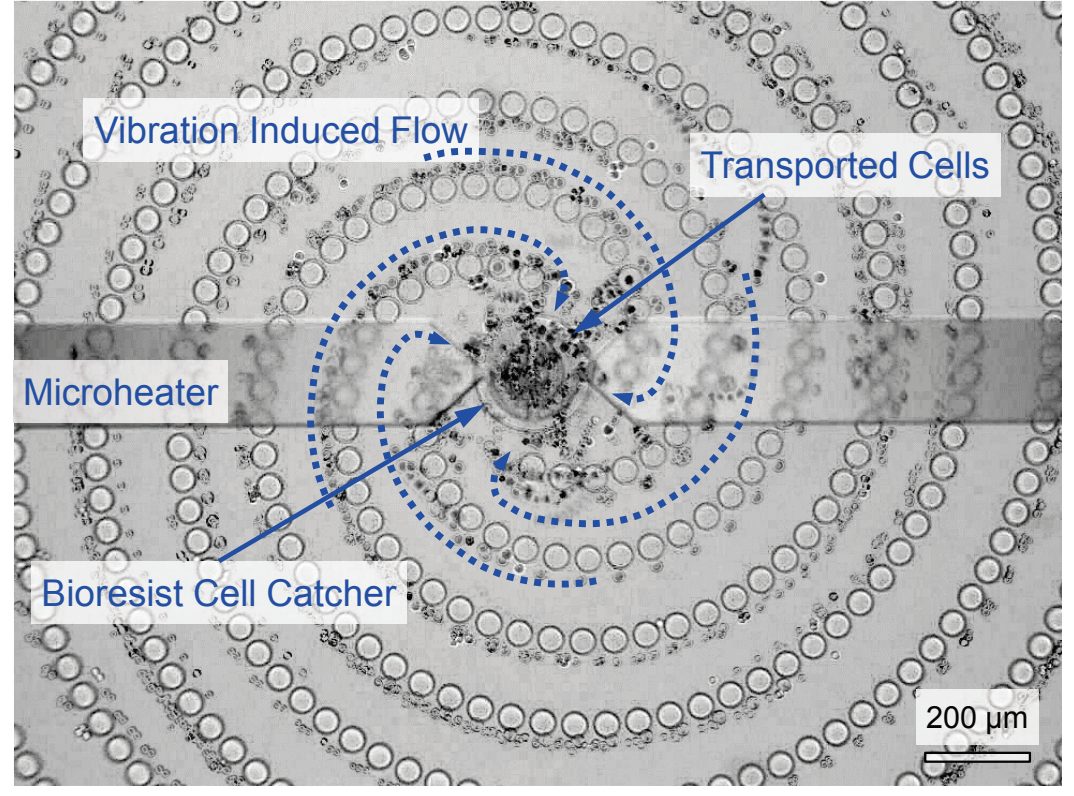

(a)

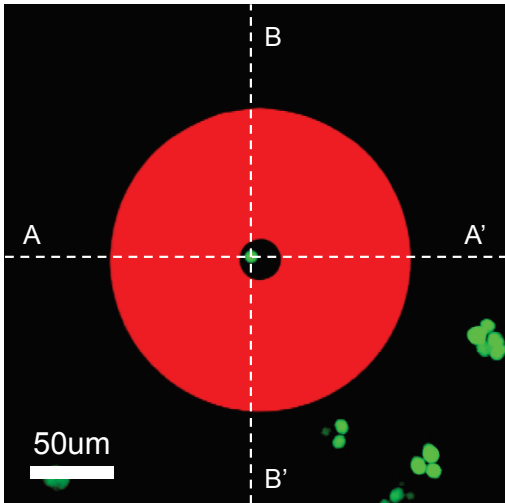

AA

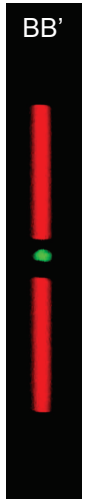

0

(c)

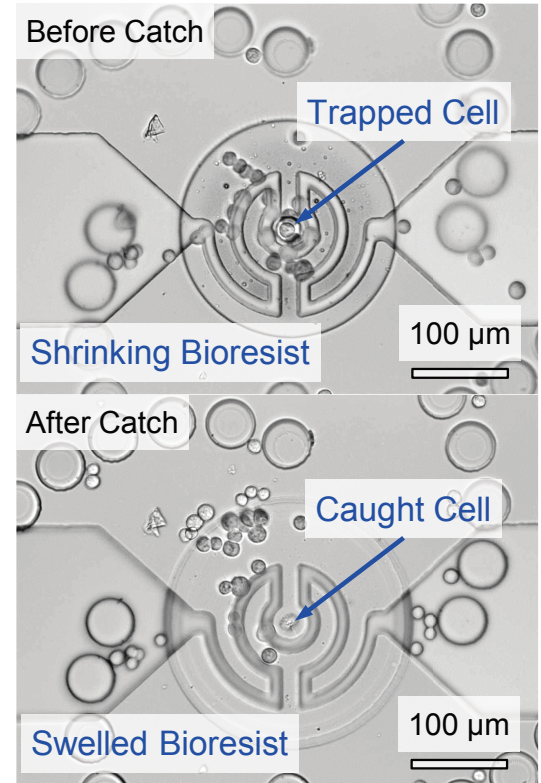

(b)

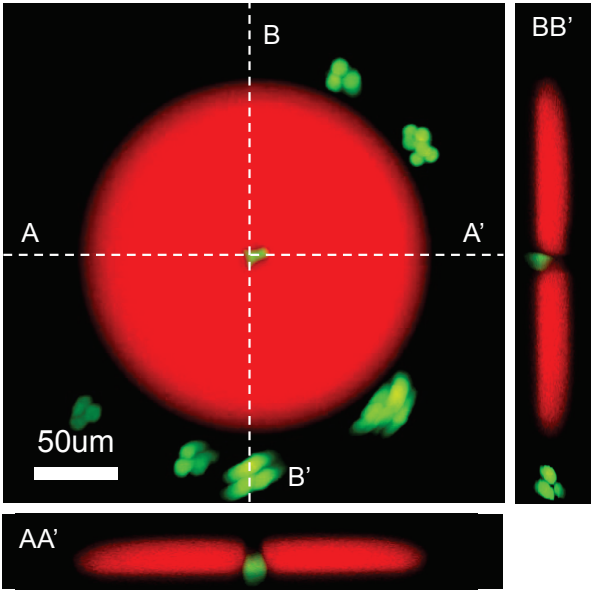

(d)

Figure 8c,d show the top and cross-sectional images (taken by a confocal fluorescent microscope) of the caught cell before and after swelling, respectively. For this observation, the bioresist was stained 
with rhodamine B (Sigma-Aldrich Co. Llc., St. Louis, MO, USA), and MDCK cells were stained with Calcein-AM (Dojindo Laboratories Co. Ltd., Tokyo, Japan). The single cell was securely caught by the swelling bioresist (Figure 8d), and it continued to be held even after washing the chip.

We performed 30 trials of single cell extraction with prepared cell suspension. Cell transport towards the hole of the single cell catcher typically took 1-3 min, and catching took 3-5 min in the trials. The whole process took less than 10 min for all trials. We succeeded in catching a single cell 30 times under microscopic observations (success rate: 100\%), and we succeeded 18 times in releasing the single cell into the culture well on commercial microtiter plate (success rate: 60\%). On the other hand, we did not succeed in catching a single cell without circular vibration with this sample concentration, even after at least 108 times trials.

We confirmed the extracted cell viability with a live/dead cell viability kit (Life technologies Co. Ltd., Taipei, Taiwan) after an $8 \mathrm{~h}$ incubation. The extracted cells were incubated in 96 well microtiter plate (Nuclon delta surface, Thermo Fisher Scientific K.K.) by using EMEM $+10 \%$ FBS in $37{ }^{\circ} \mathrm{C}, 5 \%$ $\mathrm{CO}_{2}$ environment. To confirm the effect of the extraction process, we prepared control samples and evaluate the viability. The control samples were divided separately from a same cell suspension for the experimental samples. The control samples were stored under the same condition with the experimental samples $\left(4{ }^{\circ} \mathrm{C}\right)$ during the experiments. After the experiments, approximately $4 \mathrm{~h}$ later after trypsin treatment in the first step, the control samples were incubated at the same condition as the experimental samples. Eleven cells (61\% of extracted cells) indicated live signals, and 7 cells (39\% of extracted cells) indicated dead signals. These values are not so different from the control samples, of which $71 \%$ were live and $29 \%$ were dead $(N=96)$. Therefore, the cell damage by the proposed single cell extraction method is not significant.

\section{Conclusions}

We proposed a single cell extraction chip with an open structure by utilizing vibration-induced flow and a single cell catcher composed of a thermo-responsive gel pattern. By applying circular vibration to the spiral micropillar array on the chip, whirling flow was induced and target cells were transported to the single cell catcher at the center of the spiral. The single cell catcher can be shrunk and swelled by the heating of a microheater, and the swelled catcher can catch the single cell transported by the vibration-induced flow. Finally, we conducted a single cell extraction with the proposed system and obtained a $60 \%$ success rate, of which $61 \%$ of the cells were live after incubation. Thus, the effectiveness of the on-chip single cell extraction was confirmed.

\section{Supplementary Materials}

Supplementary materials can be accessed at: http://www.mdpi.com/2072-666X/5/3/681/s1.

\section{Acknowledgments}

This study was financially supported by Grant-in-Aid for JSPS Fellows. The authors also thank Nissan Chemical Industries, Ltd. for providing some materials. 


\section{Author Contributions}

Takeshi Hayakawa, Shinya Sakuma and Fumihito Arai are equally contributed to this work. Takeshi Hayakawa, Shinya Sakuma and Fumihito Arai performed conception and design of the study, collection of data, analysis and interpretation of data, drafting of the manuscript and critical revision of the manuscript for important intellectual content. Takeshi Fukuhara performed design of the study and critical revision of the article for important intellectual content. Yoshiyuki Yokoyama performed design of the study. All authors read and approved the final manuscript.

\section{Conflicts of Interest}

The authors declare no conflict of interest.

\section{References}

1. Wang, D.; Bodovitz, S. Single cell analysis: the new frontier in 'omics'. Trends Biotechnol. 2010, 28, 281-290.

2. Narsinh, K.H.; Sun, N.; Sanchez-Freire, V.; Lee, A.S.; Almeida, P.; Hu, S.; Jan, T.; Wilson, K.D.; Leong, D.; Rosenberg, J.; Yao, M.; Robbins, R.C.; Wu, J.C. Single cell transcriptional profiling reveals heterogeneity of human induced pluripotent stem cells. J. Clin. Investig. 2011, $121,1217-1221$.

3. Hulett, H.R.; Bonner, W.A.; Barrett, J.; Herzenberg, L.A. Cell sorting: Automated separation of mammalian cells as a function of intracellular fluorescence. Science 1969, 166, 747-749.

4. Bonner, W.A.; Hulett, H.R.; Sweet, R.G.; Herzenberg, L.A. Fluorescence activated cell sorting. Rev. Sci. Instrum. 1972, 43, 404-409.

5. Huh, D.; Gu, W.; Kamotani, Y.; Grotberg, J.B.; Takayama, S. Microfluidics for flow cytometric analysis of cells and particles. Physiol. Meas. 2005, 26, R73-R98.

6. Fu, A.Y.; Spence, C.; Scherer, A.; Arnold, F.H.; Quake, S.R. A microfabricated fluorescence-activated cell sorter. Nat. Biotechnol. 1999, 17, 1109-1111.

7. Yamada, M.; Nakashima, M.; Seki, M. Pinched flow fractionation: Continuous size separation of particles utilizing a laminar flow profile in a pinched microchannel. Anal. Chem. 2004, $76,5465-5471$.

8. Mach, A.J.; Kim, J.H.; Arshi, A.; Hur, S.C.; Carlo, D.D. Automated cellular sample preparation using a centrifuge-on-a-chip. Lab Chip 2011, 11, 2827-2834.

9. Tan, W.H.; Takeuchi, S. A trap-and-release integrated microfluidic system for dynamic microarray applications. Proc. Natl. Acad. Sci. USA 2007, 104, 1146-1151.

10. Arai, F.; Ichikawa, A.; Fukuda, T.; Katsuragi, T. Isolation and extraction of target microbes using thermal sol-gel transformation. Analyst 2003, 128, 547-551.

11. Arai, F.; Ng, C.; Maruyama, H.; Ichikawa, A.; El-Shimy, H.; Fukuda, T. On chip single-cell separation and immobilization using optical tweezers and thermosensitive hydrogel. Lab Chip 2005, 5, 1399-1403.

12. Ashkin, A.; Dziedzic, J.M. Optical trapping and manipulation of viruses and bacteria. Science 1987, 235, 1517-1520. 
13. Arai, F.; Ichikawa, A.; Ogawa, M.; Fukuda, T.; Horio, K.; Itoigawa, K. High-speed separation system of randomly suspended single living cells by laser trap and dielectrophoresis. Electrophoresis 2001, 22, 283-288.

14. Eriksson, E.; Sott, K.; Lundqvist, F.; Sveningsson, M.; Scrimgeour, J.; Hanstorp, D.; Goksör, M.; Granéli, A. A microfluidic device for reversible environmental changes around single cells using optical tweezers for cell selection and positioning. Lab Chip 2010, 10, 617-625.

15. Pethig, R. Review article-Dielectrophoresis: Status of the theory, technology, and applications. Biomicrofluidics 2010, 4, 022811.

16. Chiou, P.Y.; Ohta, A.T.; Wu, M.C. Massively parallel manipulation of single cells and microparticles using optical images. Nature 2005, 436, 370-372.

17. Voldman, J.; Gray, M.L.; Toner, M.; Schmidt, M.A. A microfabrication-based dynamic array cytometer. Anal. Chem. 2002, 74, 3984-3990.

18. Ding, X.; Li, P.; Lin, S.C.S.; Stratton, Z.S.; Nama, N.; Guo, F.; Slotcavage, D.; Mao, X.; Shi, J.; Costanzoa, F.; Huang, T.J. Surface acoustic wave microfluidics. Lab Chip 2013, 13, 3626-3649.

19. Yeo, L.Y.; Friend, J.R. Ultrafast microfluidics using surface acoustic waves. Biomicrofluidics 2009, 3, 012002 .

20. Holtsmard, J.; Johnsen, I.; Sikkeland, T.; Skavlem, S. Boundary layer flow near a cylindrical obstacle in an oscillating imcompressible fluid. J. Acoust. Soc. Am. 1945, 26, 26-39.

21. Wand, C.; Drachman, B. The steady streaming generated by a vibrating plate parallel to a fixed plate. Appl. Sci. Res. 1982, 39, 55-68.

22. Lutz, B.R.; Chen, J.; Schwartz, D.T. Hydrodynamic tweezers: 1. Noncontact trapping of single cells using steady streaming microeddies. Anal. Chem. 2006, 78, 5429-5435.

23. Hagiwara, M.; Kawahara, T.; Arai, F. Local streamline generation by mechanical oscillation in a microfluidic chip for noncontact cell manipulations. Appl. Phys. Lett. 2012, 101, 074102.

24. Yokoyama, Y.; Umezaki, M.; Kishioka, T.; Tamiya, E.; Takamura, Y. Micro- and nano-fabrication of stimulus-responsive polymer using nanoimprint lithography. J. Photopolym. Sci. Technol. 2011, $24,63-70$.

25. Ito, K.; Sakuma, S.; Yokoyama, Y.; Arai, F. On-chip gel-valve using photoprocessable thermoresponsive gel. ROBOMECH J. 2014, doi:10.1186/s40648-014-0005-8.

(C) 2014 by the authors; licensee MDPI, Basel, Switzerland. This article is an open access article distributed under the terms and conditions of the Creative Commons Attribution license (http://creativecommons.org/licenses/by/3.0/). 